\title{
Early prediction of response to radiotherapy and androgen-deprivation therapy in prostate cancer by repeated functional MRI: a preclinical study
}

\author{
Kathrine Røe ${ }^{1,2^{*}}$, Manish Kakar ${ }^{1}$, Therese Seierstad ${ }^{1}$, Anne H Ree ${ }^{2,3}$ and Dag R Olsen ${ }^{4}$
}

\begin{abstract}
Background: In modern cancer medicine, morphological magnetic resonance imaging (MRI) is routinely used in diagnostics, treatment planning and assessment of therapeutic efficacy. During the past decade, functional imaging techniques like diffusion-weighted (DW) MRI and dynamic contrast-enhanced (DCE) MRI have increasingly been included into imaging protocols, allowing extraction of intratumoral information of underlying vascular, molecular and physiological mechanisms, not available in morphological images. Separately, pre-treatment and early changes in functional parameters obtained from DWMRI and DCEMRI have shown potential in predicting therapy response. We hypothesized that the combination of several functional parameters increased the predictive power.
\end{abstract}

Methods: We challenged this hypothesis by using an artificial neural network (ANN) approach, exploiting nonlinear relationships between individual variables, which is particularly suitable in treatment response prediction involving complex cancer data. A clinical scenario was elicited by using 32 mice with human prostate carcinoma xenografts receiving combinations of androgen-deprivation therapy and/or radiotherapy. Pre-radiation and on days 1 and 9 following radiation three repeated DWMRI and DCEMRI acquisitions enabled derivation of the apparent diffusion coefficient (ADC) and the vascular biomarker $K^{\text {trans }}$, which together with tumor volumes and the established biomarker prostate-specific antigen (PSA), were used as inputs to a back propagation neural network, independently and combined, in order to explore their feasibility of predicting individual treatment response measured as 30 days post-RT tumor volumes.

Results: ADC, volumes and PSA as inputs to the model revealed a correlation coefficient of $0.54(p<0.001)$ between predicted and measured treatment response, while $K^{\text {trans }}$, volumes and PSA gave a correlation coefficient of 0.66 ( $p<0.001)$. The combination of all parameters (ADC, $K^{\text {trans }}$, volumes, PSA) successfully predicted treatment response with a correlation coefficient of $0.85(p<0.001)$.

Conclusions: We have in a preclinical investigation showed that the combination of early changes in several functional MRI parameters provides additional information about therapy response. If such an approach could be clinically validated, it may become a tool to help identifying non-responding patients early in treatment, allowing these patients to be considered for alternative treatment strategies, and, thus, providing a contribution to the development of individualized cancer therapy.

Keywords: artificial neural network, back propagation neural network, diffusion weighted magnetic resonance imaging, dynamic contrast-enhanced magnetic resonance imaging, prostate cancer, androgen-deprivation therapy, radiotherapy

\footnotetext{
* Correspondence: Kathrine.Roe@rr-research.no

'Department of Radiation Biology, Institute for Cancer Research, The Norwegian Radium Hospital, Oslo University Hospital, PO Box 4953 Nydalen, 0424 Oslo, Norway

Full list of author information is available at the end of the article
} 


\section{Background}

Prostate cancer (PCa) is a disease characterized by biologically heterogeneous behaviour. Treatment of $\mathrm{PCa}$ is controversial, with no established consensus on screening or diagnostic tests for pre-treatment evaluation of $\mathrm{PCa}$ aggressiveness $[1,2]$. Consequently, the ability to differentiate between low-risk and high-risk patients and the need for and appropriateness of treatment at any stage of the disease, remains a difficult issue. Whereas low-risk PCa patients are faced with problems associated with over-treatment, high-risk PCa patients might be suffering from under-treatment and high frequency of recurrence. Thus, PCa represents a disease in which early prediction of ultimate therapeutic efficacy is critical, but so far has been challenging to achieve.

Prediction of individual therapy response is critically dependent on the ability to quantify tumor heterogeneity and heterogeneous response of tumors with otherwise identical clinical prognostic factors. Established, non-invasive methods that effectively evaluate the heterogeneous therapy responses are elusive in clinical practice. Radiological response to treatment is most commonly quantified by measuring the tumor diameter in one or two directions. However, functional magnetic resonance imaging (MRI) techniques, like diffusion-weighted (DW) MRI and dynamic contrastenhanced (DCE) MRI, are promising and have opened for repeated in vivo assessment of biomarkers from underlying vascular, molecular and physiological processes in individual tumors. DWMRI depicts the local microstructural characteristics of water diffusion, can be quantified by calculating the apparent diffusion coefficient (ADC), and enables detection of microscopic changes in tissue structure and physiology $[3,4]$. Further, by tracking the entrance of a diffusible contrast agent from the tumor vasculature and into the extravascular, extracellular space, DCEMRI allows deduction of the vascular biomarker $K^{\text {trans }}$ [5], which may be of particular importance in clinical response monitoring of increased or inhibited angiogenesis. $K^{\text {trans }}$ has also been shown to reflect tumor oxygenation status, which is an important factor for successful radiotherapy (RT) outcome [6]. Alterations in functional imaging parameters have been shown to precede tumor volume reductions, enabling identification of good and poor responders at an early time-point, and thus, facilitation of individualized treatment schedules [6-13]. These functional MRI techniques are now increasingly becoming in routine use in many radiological departments, thus, the approach presented in the current study suggests a further use of these data, by exploiting them in prediction modeling together with standard clinical parameters.
Medical artificial intelligence is a methodology that potentially can support clinicians in deciding correct diagnosis, making therapeutic decisions and predicting therapeutic outcome $[14,15]$. Artificial neural networks (ANNs) are attractive analytical tools in medicine due to their ability to learn from historical examples, analyze non-linear data and being able to generalize a model to independent data. ANNs are inspired by the biological nervous system and consist of interconnected processes utilizing parallel computations, analogous to the biological neurons being the brain's processing units. There are numerous ANN methods, however, this study concentrates on the back propagation neural network (BPNN) approach. This method was originally described by Rumelhart et al [16], and has become one of the most popular ANN algorithms in medicine, due to the demonstration of high prediction outcomes in a range of medical applications, which also inspired us to implement and test this approach. The BPNN architecture consists of many identical nodes, or neurons, mainly consisting of nonlinear activation functions. The nodes are interconnected by weights, representing the interneuron synapses in the brain. Further, the BPNN architecture is divided into three layers; input, hidden and output layers. The input layer feeds information into the network, while the nodes in the hidden layers and output layers process the information. The nodes in the hidden layer do not have predefined initial values, but do allow complex relationships between input and output nodes to develop. The training process consists of forward and backward propagation of signals. In the forward training process, input data are forwardly propagated in the network while known output parameters are kept in the output nodes to compare the results generated by the network. In the back propagation training phase, the respective differences (errors) are used to change the interconnecting weights by using a gradient learning algorithm by back propagating the errors [16].

ANNs have previously been applied on PCa patient data in order to predict treatment outcome based on clinical parameters like tumor volume, prostate-specific antigen (PSA), the primary and regional nodal extent of the tumor and the absence or presence of metastases (TNM classification), biopsy Gleason score and age as input parameters [14,17-21]. In the study by Gulliford $e t$ al [17], volume, PSA and tumor stage were used as inputs. Although the results from this study unveiled a predictive potential, both the sensitivity and the specificity were low (sensitivity; 66.8 - 70.2\%, specificity; 52.7 64.2\%). Further, Stephan et al [21] performed a study where PSA was used as input to an ANN approach in order to investigate whether such a model could differentiate between $\mathrm{PCa}$ and benign prostatic disease. Also 
in this study the specificity was low; median values $62.1 \%$ and $45.5 \%$, for $90 \%$ and $95 \%$ sensitivity, respectively. We hypothesize that the addition of functional magnetic resonance imaging (MRI) parameters into a prediction model might provide valuable intratumoral information that, in addition to the established clinical parameters, will contribute to improve the prediction of therapeutic efficacy.

To explore whether the combined use of pre-treatment and early therapy-induced changes in functional MRI parameters increases the prediction of therapeutic response, we elicited a clinical scenario by using human, androgen-sensitive prostate carcinoma xenografts receiving RT and/or androgen-deprivation therapy (ADT). Functional MRI parameters were derived after three repeated DWMRI and DCEMRI sessions, and together with volumes and PSA measurements, these parameters were independently and combined used as inputs to a BPNN in order to explore their feasibility of predicting treatment response measured as 30 days post-RT tumor volumes.

\section{Methods}

A schematic synopsis of the experiment is provided in Figure 1.

\section{Animals, xenografts and treatment}

Male, sexually mature BALB/c nude mice (30 - 35 g, 6 8 weeks old) were subcutaneously (s.c.) implanted with

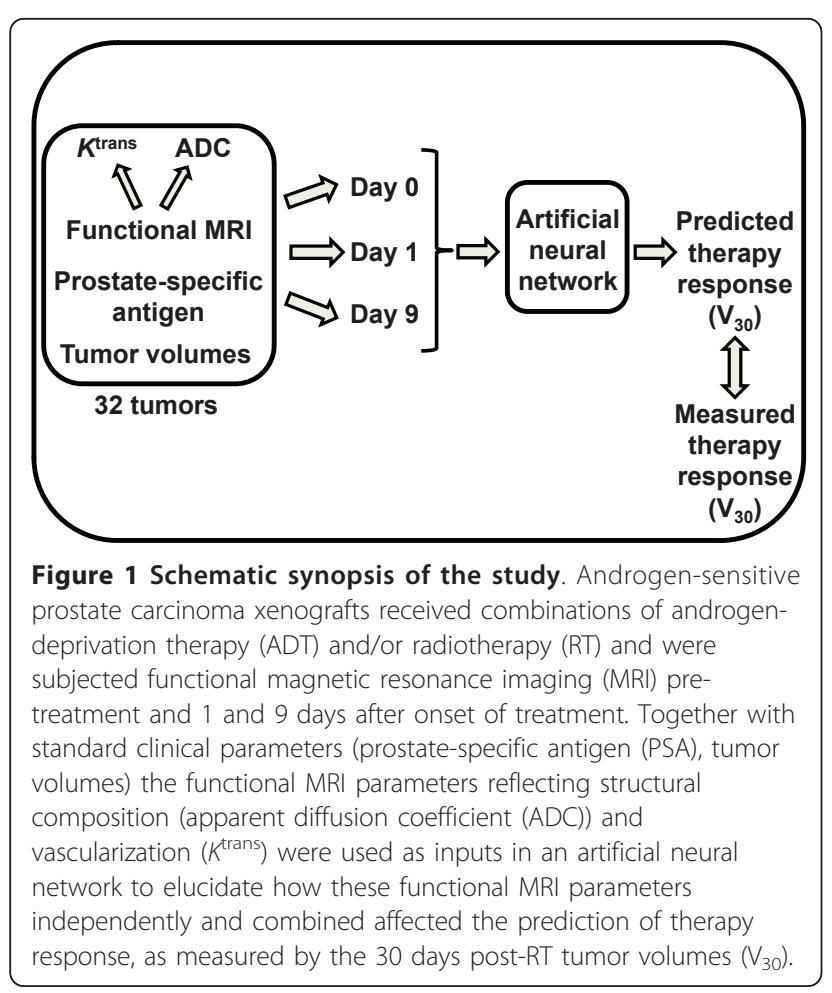

$\sim(2 \times 2 \times 2) \mathrm{mm}^{3}$ tumor tissue from the human, androgen-sensitive CWR22 xenograft. Procedures for implantation of xenografts are previously described [22]. All animal experiments were performed according to protocols approved by the animal care and use committee.

Animals were included in the experiment when their shortest tumor diameter reached $8 \mathrm{~mm}$. Androgendeprived CWR22 xenografts (CWR22-cas) were obtained by surgical castration of animals bearing CWR22 xenografts at a shortest tumor diameter of 13 $\mathrm{mm}$; the animals were included in the experiment when CWR22-cas xenografts had regressed to a shortest diameter of $8 \mathrm{~mm}$. The time from castration to inclusion was $36 \pm 4$ days. Totally 32 animals (4 groups of 8 animals) were used; CWR22 control, CWR22 irradiation, CWR22-cas control and CWR22-cas irradiation. At inclusion, animals were subjected to a pre-treatment (day 0) MRI before tumors in the irradiation groups received a single-dose of 15 Gy from a ${ }^{60}$ Co source (Mobaltron 80, TEM Instruments, Crawley, UK) with a dose rate of $0.8 \mathrm{~Gy} / \mathrm{min}$. At day 1 and day 9 repeated MRIs were performed of all animals.

Anesthesia was provided as s.c. injections of a mixture of $2.4 \mathrm{mg} / \mathrm{ml}$ tiletamine and $2.4 \mathrm{mg} / \mathrm{ml}$ zolazepam (Zoletil vet, Virbac Laboratories, Carros, France), 3.8 $\mathrm{mg} / \mathrm{ml}$ xylazine (Narcoxyl vet, Roche, Basel, Switzerland), and $0.1 \mathrm{mg} / \mathrm{ml}$ butorphanol (Torbugesic, Fort Dodge Laboratories, Fort Dodge, IA), diluted 1:5 in sterile water. A dose of $50 \mu \mathrm{l} / 10 \mathrm{~g}$ was given prior to irradiation and $75 \mu \mathrm{l} / 10 \mathrm{~g}$ before MRI and castration. Castrated animals received analgesia as $0.1 \mathrm{mg} / \mathrm{kg}$ s.c. injections of buprenorphine (Temgesic; Schering-Plough, Brussels, Belgium).

\section{MRI acquisition and analysis}

MRI was acquired at day 0 (pre-RT), day 1 and day 9, using a 1.5 T GE Signal LS scanner (GE Medical Systems, Milwaukee, WI). Animals were imaged using an MRI mouse coil [23], while the temperature was maintained at $38^{\circ} \mathrm{C}$. First, the tumor was localized using axial fast spin-echo (FSE) T2-weighted (T2W) images (echo time $\left(\mathrm{TE}_{\text {eff }}\right)=85 \mathrm{~ms}$, repetition time $(\mathrm{TR})=4000 \mathrm{~ms}$, echo train length $(\mathrm{ETL})=16$, image matrix $(\mathrm{IM})=256$ $\times 256$, field-of-view $(\mathrm{FOV})=4 \mathrm{~cm}$, slice thickness $(\mathrm{ST})$ $=2 \mathrm{~mm}$ ). Second, diffusion-weighted images (single shot FSE; $\mathrm{TE}_{\text {eff }}=78.8 \mathrm{~ms} ; \mathrm{TR}=5000 \mathrm{~ms} ; \mathrm{FOV}=14 \mathrm{~cm} ; \mathrm{IM}$ $=128 \times 128 ; \mathrm{ST}=2 \mathrm{~mm}$; interslice gap $=1 \mathrm{~mm}$; bvalues $=0$ and $100 \mathrm{~s} / \mathrm{mm}^{2}$ ) were acquired with the fol-

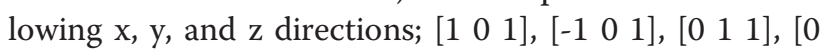
$\left.\begin{array}{ll}1 & -1\end{array}\right],\left[\begin{array}{lll}1 & 1 & 0\end{array}\right]$ and $\left[\begin{array}{lll}-1 & 1 & 0\end{array}\right]$. An axial FSE T2W sequence with identical FOV as the DWMRI was obtained for post-processing image analysis purposes. Third, the DCEMRI acquisitions were obtained as described 
elsewhere [22]. Briefly, a catheter attached to a cannula with saline-diluted Gd-DTPA (Magnevist ${ }^{\mathbb{B}}$, Schering, Berlin, Germany) was inserted into the tail vein. Dynamic T1-weighted (T1W) imaging was acquired by performing 20 minutes of dynamic fast spoiled gradientrecalled (FSPGR) imaging after the initial 5 pre-contrast images and the 3 seconds injection of the contrast agent. Time resolution was 12 seconds and the voxel size was $0.23 \times 0.47 \times 2 \mathrm{~mm}^{3}$. Proton density images were acquired prior to and after DCEMRI to allow quantification of the concentration of Gd-DTPA [24]. The vascular input function (VIF) needed in quantitative post-processing image analysis was VIF $=3.57 \pm 0.34$ $\mathrm{mM}\left(\exp \left(\left(-0.025 \pm 0.005 \mathrm{~s}^{-1}\right) \mathrm{t}\right)\right)+1.45 \pm 0.15 \mathrm{mM}(\exp$ $\left.\left(\left(-0.0074 \pm 0.0036 \mathrm{~s}^{-1}\right) \mathrm{t}\right)\right)(22)$.

Post-processing DWMRI analysis was performed in nICE (Nordic NeuroLab, Bergen, Norway). Isotropic ADC maps were calculated voxel-wise using a monoexponential approach, allowing determination of mean tumor ADCs after transferring tumor region-of-interests (ROIs) delineated in T2W MR images to the ADC maps. DCEMRI analysis was executed in IDL (Interactive Data Language v6.2, Research Systems Inc., Boulder, $\mathrm{CO})$. ROIs were traced in post-contrast T1W images, before contrast enhancement curves from individual voxels were fitted to the kinetic model of Tofts [5], allowing voxel-wise and mean tumor estimation of the vascular biomarker $K^{\text {trans }}\left(\mathrm{s}^{-1}\right)$.

\section{PSA}

Blood samples from all animals at days 0, 1 and 9 were obtained and allowed to coagulate before being centrifuged and stored at $-80^{\circ} \mathrm{C}$ until analysis. Free and total PSA were assayed by the fluoroimmunonometric AutoDELFIA ProStatus ${ }^{\mathrm{TM}}$ PSA Free/Total kit (PerkinElmer Life and Analytical Sciences, Wallac Oy, Turku, Finland).

\section{Treatment response monitoring}

From the day of implantation until day 30 post-irradiation, tumor volumes were estimated from caliper measurements by using the formula (length $\times$ length $\times$ width)/2, with length being the longest diameter across the tumor and width the corresponding perpendicular.

\section{ANN simulations}

Tumor volumes (V), PSA, ADC and $K^{\text {trans }}$ acquired pretreatment and early in treatment course were normalized to the individual baseline (day 0) measurement (Figure 2) and used as inputs to a BPNN to explore whether these parameters could predict treatment response, as measured by individual tumor volumes 30 days post-irradiation $\left(\mathrm{V}_{30}\right)$. Additionally, four categorical (binary encoded) variables representing treatment groups were used in all simulations. The BPNN repeatedly adjusted the weights of the network and the threshold of each neuron according to a criterion that the cost function minimized. The cost function was a root mean squared error (RMSE) between the target outputs and the actual outputs of the network. The two steps of the learning process included:

a) Forward propagation. The value of the calculated output, $y_{j}$, was compared to the actual output, $O_{j}$, before the output differences were inserted into the error function $E$ defined as:

$$
E=\frac{1}{2} \sum_{i=1}^{M} \sum_{j=1}^{N}\left(O_{i j}-y_{i j}\right)^{2}
$$

where $M$ is the total number of tumor response patterns given as input to the network, $N$ is the total number of output nodes of the network, and $j$ a specific output node, given a specific pattern $i$ into the network.

b) Backward propagation. The error $E$ from equation above was back propagated by updating the weights, $w_{i j}$, using scaled conjugate gradient descents:

$$
\Delta w_{i j}^{\text {new }}=\Delta w_{i j}^{\text {old }}-\frac{\partial E}{\partial w_{i j}} \eta
$$

where $\eta(0<\eta<1)$ controlled the learning rate of the algorithm. The learning process continued until $E$ converged to a predefined value or until the maximum number of epochs was reached. An epoch is a single pass of the data through the network, i.e. the different tumor response patterns (V, PSA, ADC and/or $K^{\text {trans }}$ ) for all experimental groups through the training set, followed by the validation set and the testing set.

All ANN simulations were performed in the Matlab Neural Network Toolbox, software version 4.0.2 (The Mathworks, Inc., Natick, MA).

Three different simulations with different sets of input parameters were performed using the same BPNN architecture. For all simulations, the BPNN used six hidden layers and a sequential mode for training, while keeping $\eta=0.4$. The first simulation used a dataset consisting of numerical normalized inputs of ADC, $\mathrm{V}$ and PSA from days 0,1 and 9, and categorical variables representing treatment groups. The architecture, including hidden layers, of the neural network is illustrated in Figure 3. In the second simulation, the dataset consisted of numerical $K^{\text {trans }}, \mathrm{V}$ and PSA values from days 0,1 and 9 , in addition to the treatment groups. The last simulation included all numerical parameters (ADC, $K^{\text {trans }}, \mathrm{V}$ and PSA) and treatment groups. 


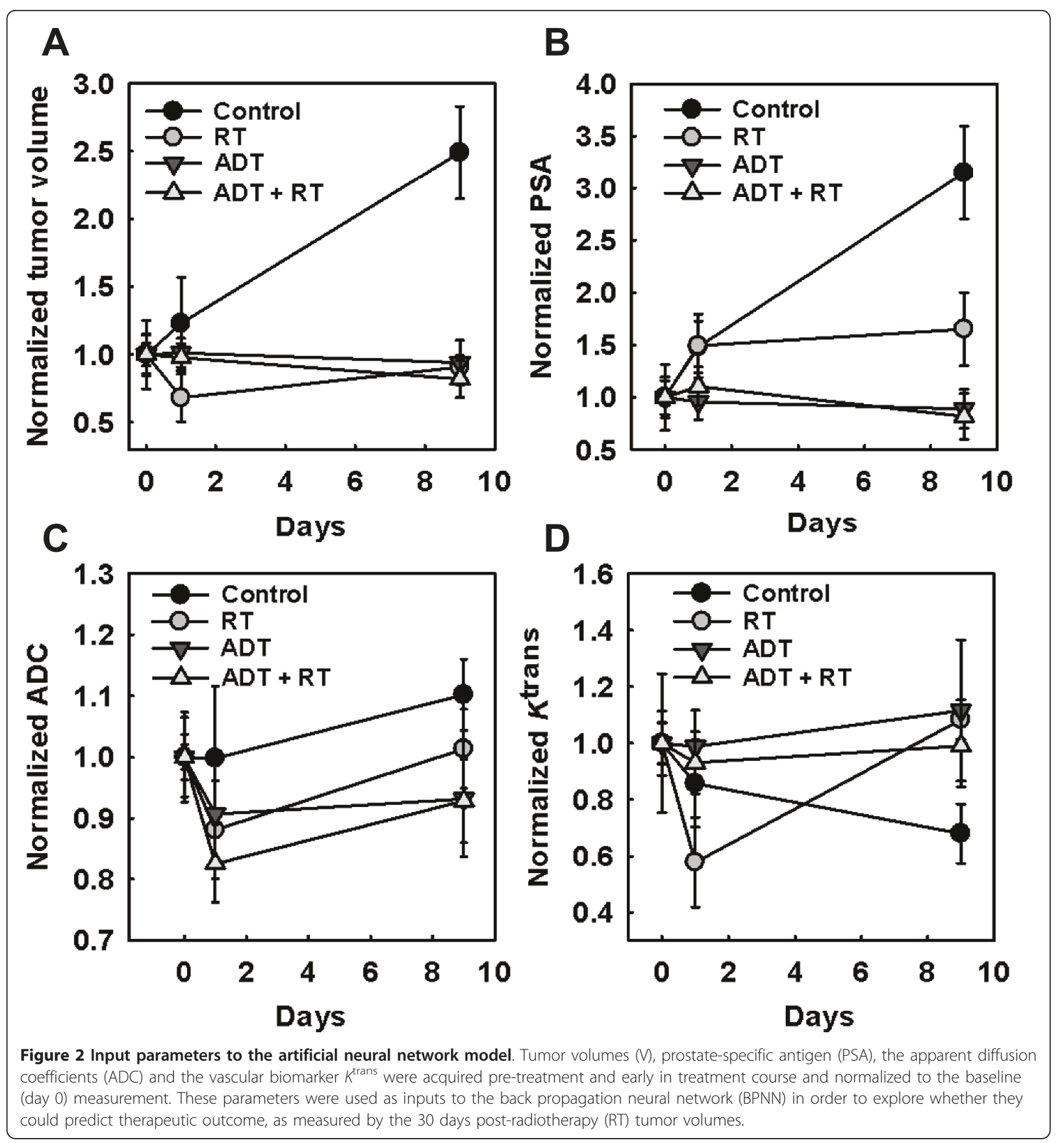

Statistical analysis

Using a significance level of 5\%, the Pearson's correlation test (SPSS 16.0, SPSS, Cary, NC) assessed whether correlations between variables were significant.

\section{Results}

Ultimate treatment response was measured as tumor volumes at day $30\left(\mathrm{~V}_{30}\right)$. Volumes of tumors in the untreated group increased with $940 \pm 91 \%$ compared to baseline (day 0 ) volumes, whereas tumors receiving radiation were $60 \pm 25 \%$ larger at the endpoint than at baseline. Androgen-deprivation alone resulted in reduction in tumor volumes by $40 \pm 9 \%$ compared to baseline, whereas tumors receiving combined androgendeprivation and radiotherapy presented a $64 \pm 5 \%$ tumor volume reduction at the experimental endpoint. 


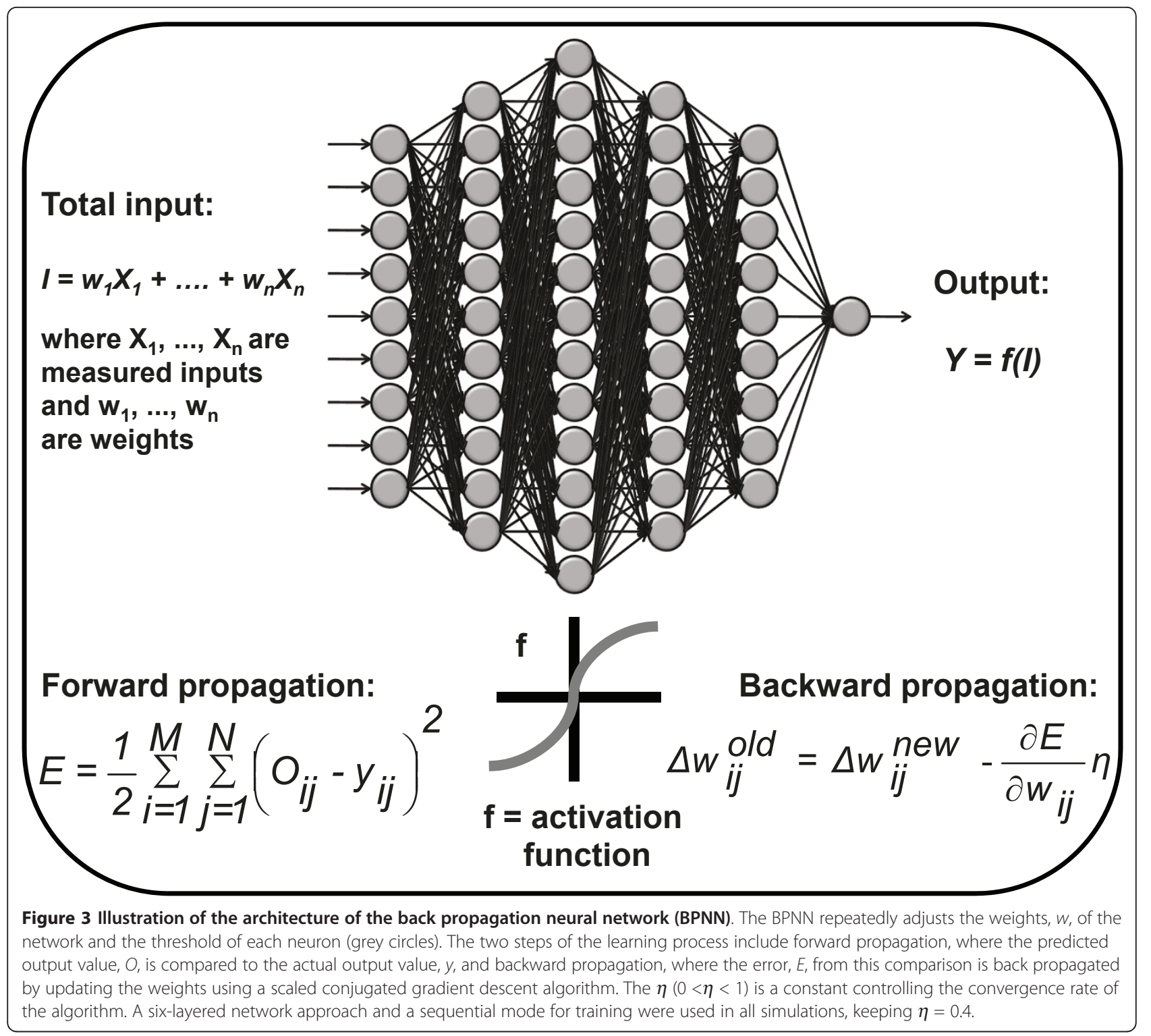

By using a BPNN with a scaled conjugate gradient learning algorithm, 50\% of the data were used for training, 25\% for validation and $25 \%$ for testing. The RMSE plot in Figure 4A shows the performance of the testing and validation of the first simulation, where normalized values of ADC, PSA and tumor volumes from days 0, 1 and 9, in addition to treatment groups (binary encoded), were used as input variables. For this simulation, the RMSE increased after 30 epochs, indicating overtraining. Thus, the optimal training for the network was found to be 30 epochs. The optimal number of epochs for the second and third simulations was decided by a similar approach as above, and found to be 49 and 69 , respectively.

The use of ADC together with tumor volumes, PSA and treatment groups as inputs to the BPNN model revealed a correlation coefficient of $0.54(\mathrm{p}<0.001)$ between predicted and measured treatment response $\left(\mathrm{V}_{30}\right)$ (Figure $\left.4 \mathrm{~B}\right)$. By replacing the ADC with $K^{\text {trans }}$, the correlation coefficient increased to 0.66 ( $\mathrm{p}<0.001$ ) (Figure 5). However, the combination of all parameters (V, PSA, ADC, $K^{\text {trans }}$ ) predicted treatment response with a correlation coefficient of 0.85 ( $\mathrm{p}<0.001)$ between predicted and measured $\mathrm{V}_{30}$ (Figure 6). This approach was superior to all other ANN simulations using the parameters independently.

\section{Discussion}

Assessment of therapeutic efficacy in $\mathrm{PCa}$ patients represents a controversial issue in clinical medicine due to the heterogeneity of the disease and its unpredictable treatment response. Although the use of ADT and/or 


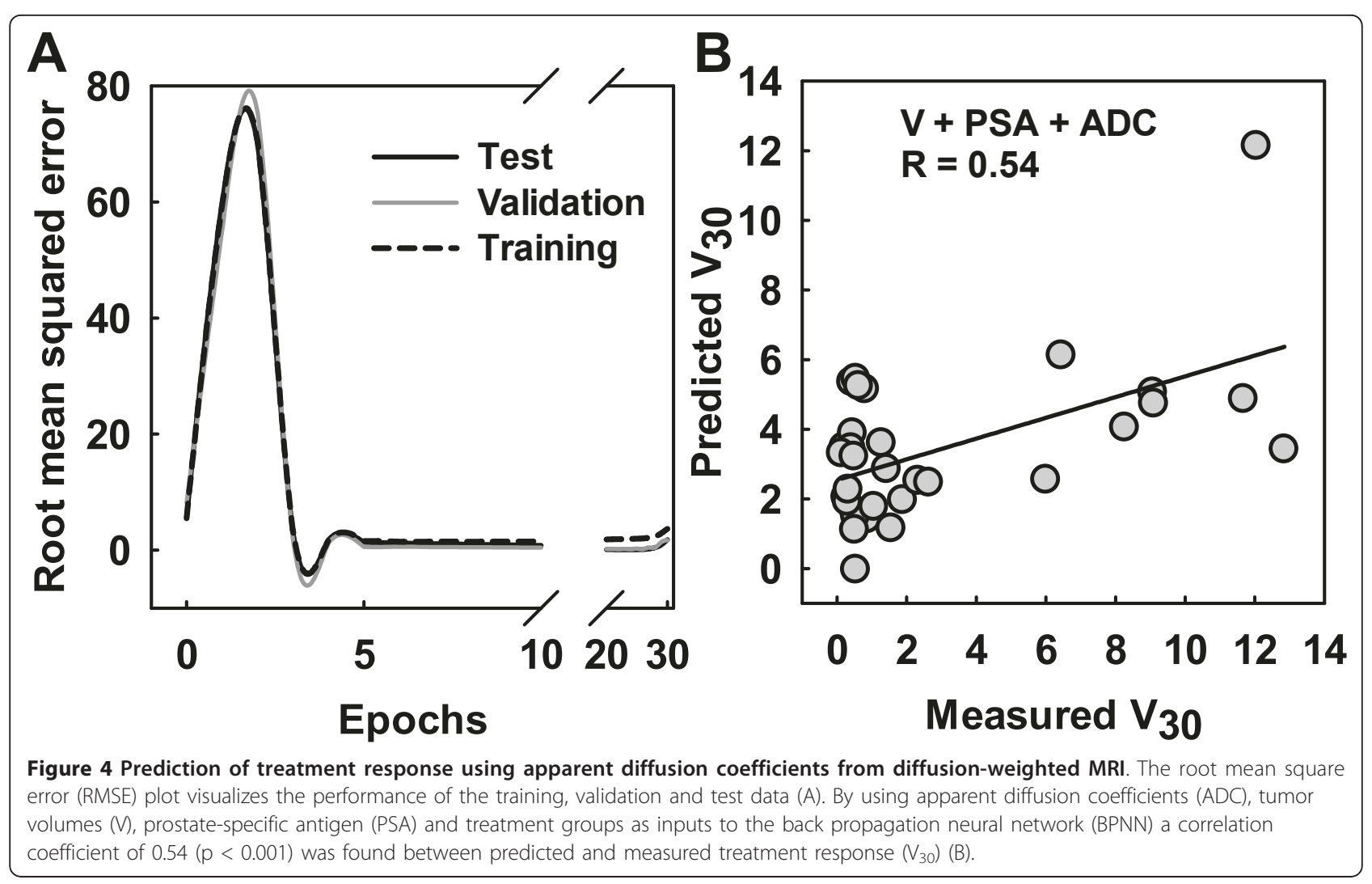

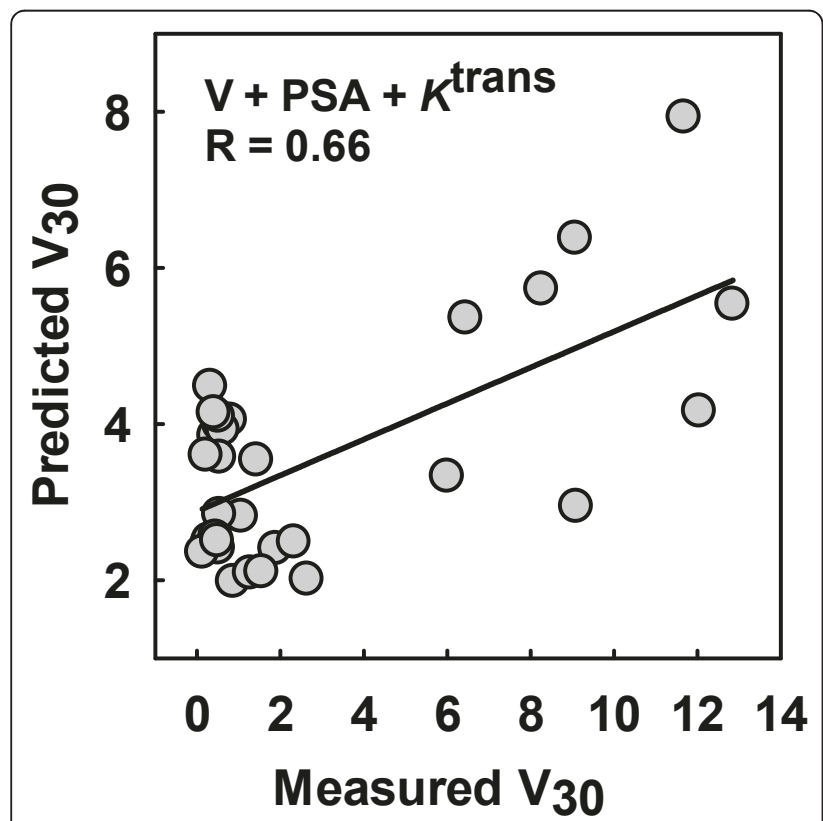

Figure 5 Prediction of treatment response using $K^{\text {trans }}$ from dynamic contrast-enhanced MRI. By using $K^{\text {trans }}$, tumor volumes $(V)$, prostate-specific antigen (PSA) and treatment groups as inputs to the back propagation neural network (BPNN) a correlation coefficient of $0.66(p<0.001)$ was found between predicted and measured treatment response $\left(V_{30}\right)$.

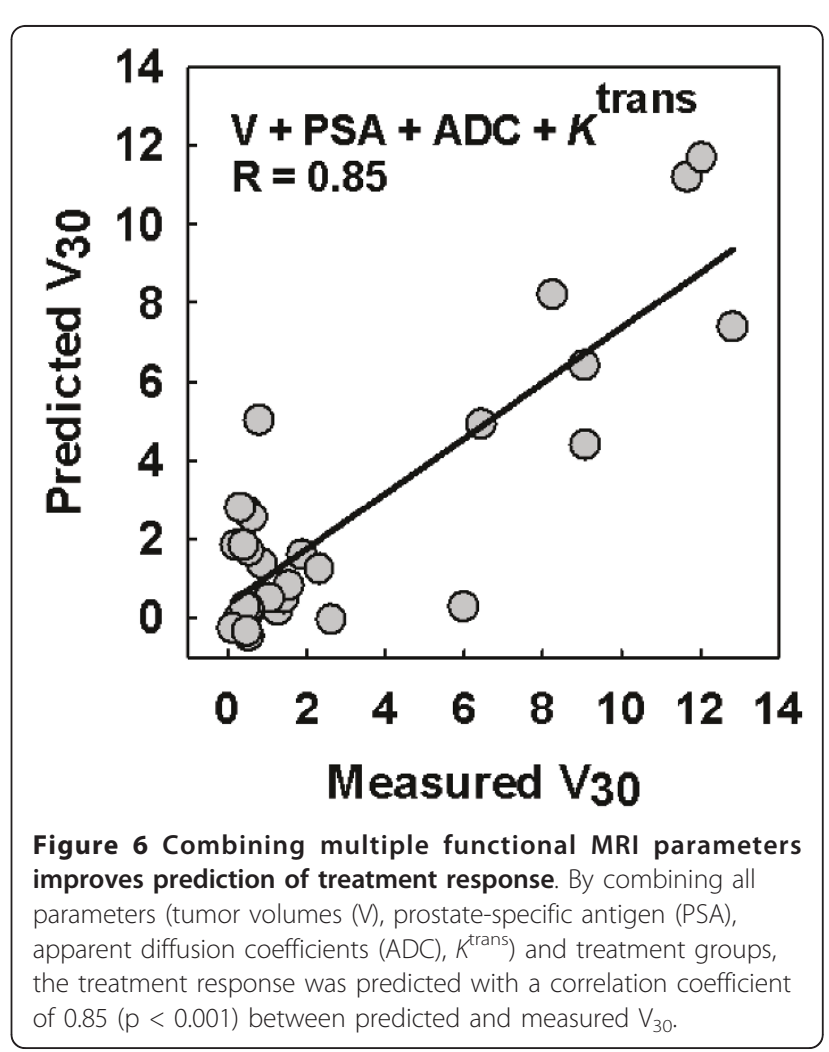


RT causes tumor regression, complete remission often fails and high-risk patients usually present recurrent disease within few years. Classic prognostic factors, like tumor volume, PSA, TNM classification and Gleason score, are those that currently guide therapy selection. However, these may be suboptimal in predicting outcome for individual patients, as these factors are not accounting for the underlying heterogeneity in vascular, molecular and physiological processes causing large variations in individual tumor responses. Thus, these factors may not enable prediction of treatment failure early in the course of treatment, when therapeutic adjustments still are feasible in terms of e.g. radiation dose escalation or alterations in concurrent therapy.

The benefits of ANNs compared to conventional regression statistics comprise the capability of being more accurate for large and complex data materials, e.g. patient data with multiple parameters from multiple measurement time-points. The artificial intelligence models of biological systems can be generated without needing assumptions about the underlying statistical distributions. Currently, in vivo imaging techniques are rapidly evolving and being extensively tested for their capability of correctly reflecting biological and physiological properties of tumor tissue. Such functional information is particularly beneficial for ANNs, since data from multiple sources effectively can be incorporated without needing knowledge on the combination of underlying biological information.

The presented results were obtained in a preclinical study in prostate cancer xenografts, and suggest that the combination of functional MRI parameters, in addition to standard clinical parameters, increases the power of predicting therapeutic outcome in prostate carcinoma after treatment with ADT and/or RT. Our two first simulations included each individual tumor's ADC from DWMRI, or $K^{\text {trans }}$ from DCEMRI, respectively, in addition to the standard clinical parameters tumor volume and PSA. The correlations between the BPNN predicted and the measured treatment response were found to be significant, but not very strong $(R=0.54$ and $R=0.66$, respectively). When we combined both the ADC and the $K^{\text {trans }}$ results in the third simulation, this gave a considerable increase in the correlation between the predicted and measured outcome $(\mathrm{R}=0.85)$, indicating that these parameters together reflect important treatment response-related information of the tumors.

Our results were obtained in a human xenograft model, thus, the next step could be to apply the same approach in a clinical setting, including parameters from functional MRI, as well as standard clinical parameters, from PCa patients receiving ADT and/or RT treatment. In the present study, the post-treatment imaging was performed at day 1 and day 9 , and these time-points are maybe not easily translated into clinical assessment of early treatment response. However, in recent years, the use of imaging modalities for early-in-treatment response assessments, for example 3 to 8 weeks after initiation of therapy, has increased, and showed potential to evaluate whether the patient respond to the chosen treatment or not. If this could be reliably measured, or predicted, from this early imaging assessment, this may help deciding whether the patient should receive intensified, or altered treatment, or possibly a reduction in unnecessary treatment. Moreover, if accounting for the five times faster metabolism in mice, 9 days would translate into approximately 6 weeks in a human, and thus, this may be a relevant time-point for treatment response evaluation, although not directly comparable. However, the results from the current study suggest a promising additional utilization of the large amounts of image data presently being acquired in hospitals. If the model is validated in clinical data, the presented methodology might become an early assay for treatment response prediction, wherein different pre-treatment and early in-treatment functional imaging parameters may be combined with standard clinical parameters in order to increase the prediction of how individual tumors respond to therapy.

Although all simulations demonstrate significant correlations between the predicted tumor volume 30 days post-RT and the measured tumor volumes, Figures $4 \mathrm{~B}$, 5 and 6 also indicate a spread in the data points. This implies that there is a probability of misclassifying the response from individual tumors, meaning that precaution should be taken if extrapolations to individuals are performed uncritically. Further, when using a BPNN, care should also be taken when training the network, in order not to under- or overtrain it [25]. Since our RMSE function (Figure 4A) from training, testing and validating the network showed curve flattening after a few training epochs, this indicated that no overtraining occurred. However, if such a model is to be applied on clinical data, the model must be rigorously validated, for example with respect to the number of layers and epochs. Patient materials will always present a larger biological heterogeneity than xenografts grown in immune-deficient mice, representing a risk for overtraining the network if the BPNN parameters are not chosen cautiously.

\section{Conclusion}

The presented results, derived from a preclinical study in prostate cancer xenografts, indicate that the combination of several functional MRI parameters obtained pre-treatment and early in the course of treatment, into an artificial neural network model, may provide additional, useful information about therapy response. If clinically 
established, this approach may help identifying nonresponding patients early during treatment course, allowing these patients to be considered for alternative treatment strategies, and, thus, providing a contribution to the development of personalized prostate cancer therapy.

\section{List of abbreviations}

ADC: apparent diffusion coefficient; ADT: androgen-deprivation therapy ANN: artificial neural network; BPNN: back propagation neural network; DCEMRI: dynamic contrast-enhanced magnetic resonance imaging; DWMRI: diffusion-weighted magnetic resonance imaging; PCa: prostate cancer; PSA: prostate specific antigen; RMSE: root mean square error; RT: radiotherapy; $V$ : volume

\section{Acknowledgements}

We thank Professor F. Saatcioglu at Department of Molecular Biosciences, University of Oslo, for providing the CWR22 xenograft model, Professor E. Paus and coworkers at the Central Laboratory, Department of Medical Biochemistry, The Norwegian Radium Hospital, Oslo University Hospital, for PSA analysis of blood samples, and department engineer Alexandr Kristian, Department of Tumor Biology, Institute for Cancer Research, The Norwegian Radium Hospital, Oslo University Hospital, for excellent assistance in animal experiments

Supported by the South-Eastern Norway Regional Health Authority (grant 2009070, to KR), the Norwegian Cancer Society (grant 80114001, to TS), and the European Union 7th Framework Programme Grant 222741 - METOXIA. Previous presentation: presented at the $21^{\text {st }}$ meeting of the European Association for Cancer Research (EACR) in Oslo, June 26 - 29, 2010.

\section{Author details}

'Department of Radiation Biology, Institute for Cancer Research, The Norwegian Radium Hospital, Oslo University Hospital, PO Box 4953 Nydalen, 0424 Oslo, Norway. ${ }^{2}$ Institute of Clinical Medicine, University of Oslo, Oslo, Norway. ${ }^{3}$ Department of Oncology, Akershus University Hospital, Lorenskog, Norway. ${ }^{4}$ University of Bergen, Bergen, Norway.

\section{Authors' contributions}

KR designed the study, carried out the animal experiments, MRI data acquisition and analysis, participated in ANN simulations and wrote the manuscript. MK developed software and performed the ANN simulations, and contributed in revision of the manuscript. TS and AHR helped in discussion of the data and revision of the manuscript. DRO participated in study design, data discussion and revision of the manuscript. All authors read and approved the final manuscript.

\section{Competing interests}

The authors declare that they have no competing interests.

Received: 1 February 2011 Accepted: 8 June 2011

Published: 8 June 2011

\section{References}

1. Mitchell RE, Chang SS: Current controversies in the treatment of high-risk prostate cancer. Curr Opin Urol 2008, 18:263-268.

2. Barry MJ: Screening for prostate cancer-the controversy that refuses to die. N Engl J Med 2009, 360:1351-1354.

3. Bammer R: Basic principles of diffusion-weighted imaging. Eur J Radiol 2003, 45:169-184.

4. Koh DM, Padhani AR: Diffusion-weighted MRI: a new functional clinical technique for tumour imaging. Br J Radiol 2006, 79:633-635.

5. Tofts PS, Brix G, Buckley DL, Evelhoch JL, Henderson E, Knopp MV, Larsson HB, Lee TY, Mayr NA, Parker GJ, Port RE, Taylor J, Weisskoff RM: Estimating kinetic parameters from dynamic contrast-enhanced T(1)weighted MRI of a diffusable tracer: standardized quantities and symbols. J Magn Reson Imaging 1999, 10:223-232.

6. Zahra MA, Hollingsworth KG, Sala E, Lomas DJ, Tan LT: Dynamic contrastenhanced MRI as a predictor of tumour response to radiotherapy. Lancet Oncol 2007, 8:63-74.
7. Seierstad T, Folkvord S, Røe K, Flatmark K, Skretting A, Olsen DR: Early changes in apparent diffusion coefficient predict the quantitative antitumoral activity of capecitabine, oxaliplatin, and irradiation in HT29 xenografts in athymic nude mice. Neoplasia 2007, 9:392-400.

8. Theilmann RJ, Borders R, Trouard TP, Xia G, Outwater E, Ranger-Moore J, Gillies RJ, Stopeck A: Changes in water mobility measured by diffusion MRI predict response of metastatic breast cancer to chemotherapy. Neoplasia 2004, 6:831-837.

9. Moffat BA, Chenevert TL, Lawrence TS, Meyer CR, Johnson TD, Dong Q, Tsien C, Mukherji S, Quint DJ, Gebarski SS, Robertson PL, Junck LR, Rehemtulla A, Ross BD: Functional diffusion map: a noninvasive MRI biomarker for early stratification of clinical brain tumor response. Proc Natl Acad Sci USA 2005, 102:5524-5529.

10. Cui Y, Zhang XP, Sun YS, Tang L, Shen L: Apparent diffusion coefficient: potential imaging biomarker for prediction and early detection of response to chemotherapy in hepatic metastases. Radiology 2008, 248:894-900.

11. Kakar M, Seierstad T, Røe K, Olsen DR: Artificial neural networks for prediction of response to chemoradiation in HT29 xenografts. Int J Radiat Oncol Biol Phys 2009, 75:506-511.

12. Kim CK, Park BK, Lee HM, Kim SS, Kim E: MRI techniques for prediction of local tumor progression after high-intensity focused ultrasonic ablation of prostate cancer. AJR Am J Roentgenol 2008, 190:1 180-1186.

13. Kurhanewicz J, Vigneron D, Carroll P, Coakley F: Multiparametric magnetic resonance imaging in prostate cancer: present and future. Curr Opin Urol 2008, 18:71-77.

14. Lisboa PJ, Taktak AF: The use of artificial neural networks in decision support in cancer: a systematic review. Neural Netw 2006, 19:408-415.

15. Ramesh AN, Kambhampati $C$, Monson JR, Drew PJ: Artificial intelligence in medicine. Ann R Coll Surg Engl 2004, 86:334-338.

16. Rumelhart $D E$, Hinton GE, Williams RJ: Learning representations by backpropagating errors. Nature 1986, 323:533-536.

17. Gulliford SL, Webb S, Rowbottom CG, Corne DW, Dearnaley DP: Use of artificial neural networks to predict biological outcomes for patients receiving radical radiotherapy of the prostate. Radiother Oncol 2004, 71:3-12.

18. Gamito EJ, Crawford ED: Artificial neural networks for predictive modeling in prostate cancer. Curr Oncol Rep 2004, 6:216-221.

19. Tewari A, Issa M, El-Galley R, Stricker H, Peabody J, Pow-Sang J, Shukla A, Waisman Z, Rubin M, Wei J, Montie J, Demers R, Johnson CC, Lamerato L, Divine GW, Crawford ED, Gamito EJ, Farah R, Naravan P, Carlson G, Menon M: Genetic adaptive neural network to predict biochemical failure after radical prostatectomy: a multi-institutional study. Mol Urol 2001, 5:163-169.

20. Finne $P$, Finne $R$, Auvinen $A$, Juusela $H$, Aro J, Määttänen L, Hakama M, Rannikko S, Tammela TL, Stenman U: Predicting the outcome of prostate biopsy in screen-positive men by a multilayer perceptron network. Urology 2000, 56:418-422.

21. Stephan C, Kahrs AM, Cammann H, Lein M, Schrader M, Deger S, Miller K, Jung K: A [-2]proPSA-based artificial neural network significantly improves differentiation between prostate cancer and benign prostatic diseases. Prostate 2009, 69:198-207.

22. Røe K, Seierstad T, Kristian A, Mikalsen LT, Mælandsmo GM, van der Kogel AJ, Ree AH, Olsen DR: Longitudinal magnetic resonance imagingbased assessment of vascular changes and radiation response in androgen-sensitive prostate carcinoma xenografts under androgenexposed and androgen-deprived conditions. Neoplasia 2010, 12:818-825.

23. Seierstad T, Røe K, Høvik B: Construction of a modified capacitive overlap MR coil for imaging of small animals and objects in a clinical wholebody scanner. Phys Med Biol 2007, 52:N513-N522.

24. Hittmair $\mathrm{K}$, Gomiscek $\mathrm{G}$, Langenberger $\mathrm{K}$, Recht $\mathrm{M}$, Imhof $\mathrm{H}$, Kramer J: Method for the quantitative assessment of contrast agent uptake in dynamic contrast-enhanced MRI. Magn Reson Med 1994, 31:567-571.

25. Geman S, Bienenstock E, Doursat R: Neural networks and the bias/ variance dilemma. Neural Comput 1992, 4:1-58.

doi:10.1186/1748-717X-6-65

Cite this article as: Røe et al: Early prediction of response to radiotherapy and androgen-deprivation therapy in prostate cancer by repeated functional MRI: a preclinical study. Radiation Oncology 2011 6:65. 